\title{
ESTUDIO DESCRIPTIVO, COMPARATIVOY CORRELACIONAL DE LAS MANIFESTACIONES PSICOPATOLÓGICAS EN NIÑOS LIMEÑOS
}

\author{
Ernesto Oliver Paredes
}

\section{RESUMEN}

El estudio está dirigido a cuantificar, los trastornos psicopatológicos de los niños menores de catorce (14) años de edad de los diferentes estratos socioeconómicos de la ciudad de Lima Metropolitana, que se han estudiado, señalando la prevalencia promedio actual, los trastornos psicopatológicos, la morbilidad correspondiente, en porcentajes y de manera general.

Palabras CLAVE: Psicopatología, salud mental infantil, niños escolares, Lima.

\section{DESCRIPTIVE, COMPARATIVE AND CORRELATIONAL STUDY OF DEMONSTRATIONS PSYCHOPATHOLOGICAL CHILDREN FROM LIMA}

\begin{abstract}
The study is aimed at quantifying, psychopathological disorders of children under fourteen (14) years of age, of different socioeconomic strata of the city of Lima, which have been studied, indicating the current average prevalence of psychopathological disorders, the corresponding disease, in percentages and in general.
\end{abstract}

KEYWORDS: Psychopathology, child mental health, school children, Lima. 


\section{Fundamentación de la investigación}

\section{Antecedentes}

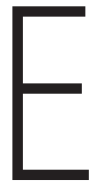

s del caso el señalar que en nuestro medio los estudios epidemiológicos de prevalencia y los de incidencia, son muy pocos y los referidos a la edad pre-escolar y escolar primaria son nimios, sobre todo en el campo de un estudio descriptivo, comparativo y de correlación, todo esto basado en la línea de investigación que durante muchos años viene realizando el autor de la presente investigación, marcando un hito, debido a la contribución que hace y a la constancia de la línea investigada, es por ello que ahora, al haber acumulado varios estudios epidemiológicos, fundamentalmente de prevalencia, es que se intenta abordar estudios de incidencia, con la descripción, comparación y correlación que sus antecedentes así la fundamentan.

Será un estudio original por cuanto los comportamientos anormales, mayormente se detectan de manera fundamental en los Servicios de Pediatría, Neurología Infantil, Psiquiatría Infantil y Psicología Infantil de los Hospitales Generales, Centros de Salud, Postas Médicas y Consultorios privados de médicos y psicólogos, así como en las Guarderías, Cunas, Nidos, Escuelas Iniciales, y en los Domicilios de los niños, pero estos hallazgos no llegan a ser consignados en un Registro Único, al cual se pueda recurrir, pues no existe, y los datos hallados se pierden, o en el mejor de los casos, a ellos no se les da la aplicación y difusión adecuadas, quedando al final en obsoletos e inservibles, pese al gran trabajo y sacrificio que significó para los estudiosos e investigadores.

Además, será la condensación de los estudios, en este caso haciendo un estudio descriptivo, comparativo y correlacional, que permita precisar los hallazgos anteriores, llevados a cabo por Oliver Paredes, Ernesto; los que ponen de manifiesto la inquietud del investigador, y/o su compromiso de ir acercándose a una realidad olvidada en el campo de la Salud Mental, y será original en el campo de la Salud Mental Infantil, en nuestro país.

Ahora se tienen como antecedentes directos, y como base de la presente investigación a los estudios de Oliver Paredes, Ernesto, así:

1. Del año 2000 denominado: "Aproximación Discreta a la Epidemiología de las Manifestaciones Psicopatológicas Infantiles, en Niños Pre-escolares del Cono Sur de Lima Metropolitana"; el cual informa de un $92.66 \%$ de morbilidad.

2. El estudio del año 2001, titulado: "Aproximación Epidemiológica a los Trastornos Psicopatológicos, en Pre-escolares de Lima Metropolitana"; el cual informa de un $89.25 \%$ de morbilidad.

3. La investigación del año 2002, Ilamada: "Aproximación Discreta al estudio de la Prevalencia de los Trastornos Psicopatológicos, en Pre-escolares de Lima Metropolitana"; el cual informa de un $86.84 \%$ de morbilidad. 
4. Oliver Paredes, Ernesto en el año del 2003, en la investigación titulada "Prevalencia de la Salud Mental, en Pre-escolares de Lima Metropolitana"; el cual informa de una morbilidad del $82.35 \%$.

5. El estudio del año 2004, denominado:"Epidemiologia del Comportamiento Anormal, en Niños de Lima Metropolitana"; el cual informa de un 78.42\% de morbilidad.

6. En el año del 2005, llamada: "Aproximación Discreta al Estudio de la Prevalencia de los Trastornos Psicopatológicos Específicos, en Niños Pre-escolares de Lima Metropolitana", en la cual informa de un $75.11 \%$ de morbilidad.

7. Oliver Paredes, Ernesto, en el 2006a, en la investigación llamada "Epidemiología: Indicadores psicopatológicos específicos, en niños limeños menores de seis años de edad", comprendiendo todos los estratos socio-económicos de manera representativa, presenta lo hallado de la manera siguiente; con morbilidad el 75\%.

8. Oliver Paredes, Ernesto, en el 2006b, en la investigación, ahora ya referida a niños en edad escolar primaria, titulada, "Aproximación a la prevalencia de los tastarnos psicopatológicos, en niños de edad escolar primaria de Lima Metropolitana", comprendiendo a los estratos socio-económicos representativos, presenta lo hallado así. Con morbilidad el $67.08 \%$.

9. Oliver Paredes, Ernesto, en el año de 2007a, en la investigación, en niños en edad escolar primaria, titulada: "Trastornos psicopatológicos en niños en edad escolar primaria de los 6 años a los 12 años de edad". Halla una morbilidad del 69.5\%.

10. Oliver Paredes, Ernesto, hace un compendio de una investigación original, no tomada en cuenta los estudios anteriores, y lo publica, en el 2007b, como un libro, que se denomina:"Psicopatología Infantil y Epidemiología Clínica: Indicadores de alteraciones psíquicas", donde encuentra una morbilidad del 75\%, para los niños pre-escolares.

11. Oliver Paredes, Ernesto, en el año del 2008, en la investigación titulada: "Indicadores generales de trastornos psicopatológicos, en niños en edad escolar primaria". Halla una morbilidad del $89.13 \%$.

12. En el año del 2009, realiza otro trabajo similar que denomina"Indicadores específicos de alteraciones psíquicas en niños de edad escolar primaria", donde encuentra una morbilidad del $83 \%$.

13. En el año del 2010, sucede un hecho semejante en el trabajo "Prevalencia psicopatológica en niños limeños menores de 13 años de edad", donde encuentra una prevalencia del $82.88 \%$.

\section{Justificación de la investigación}

Como lo señala la literatura especializada, en principio, y luego la práctica clínica infantil, sobre el tema referido al estudio de los métodos y procedimientos empleados en el diagnóstico y tratamiento en los campos de la psiquiatría infantil, psicopatología infantil y psicología infantil, se requieren de sólidos conocimientos, de la psicopatología infantil y de 
los fundamentos de la probable causalidad, así como de conocer cuáles son los problemas más frecuentes, que originan el envío a tratamiento por el especialista de determinados niños; y cuando esto acontece, el abordaje al estudio de la familia se hace básico e indispensable, pero todo este planteamiento, tiene que estar fundado en el conocimiento previo, de los aspectos epidemiológicos, primero probablemente causales de índole cualitativa e inmediatamente los de carácter cuantitativo, donde la prevalencia (el número de casos de una enfermedad existente en una población, en un tiempo determinado), así como los aspectos de la morbilidad que configuran los comportamientos anormales infantiles, y dentro de ellos los correspondientes a la edad pre-escolar y escolar, que es motivo de interés del presente estudio, el cual es una saga que se inició en el año 2000, pareciendo que el presente estudio, aportaría la aproximación discreta más cercana a la realidad de lo que acontece en esta edad evolutiva, por ser en este período donde aparecen las primeras manifestaciones, que en el futuro podrían convertirse en serios problemas psicopatológicos y/o psiquiátricos a tratar, es por ello que este estadio del desarrollo, se vuelve inevitablemente el más importante, y todos los datos epidemiológicos de prevalencia se constituyen en una guía válida y un derrotero hacia un mejor afronte preventivo al inicio y diagnóstico y terapéutico posterior, para el especialista en el campo de la salud mental infantil; así como para el interesado en este grupo de edad, o de la etapa de la infancia de manera general.

La actual población de nuestro país, por su estructura de edad es "relativamente joven"; sin embargo, considerando las tendencias de la dinámica demográfica, en el año 2020 se produciría un cambio importante en su composición.

Los menores de 15 años perderán su importancia relativa, llegando al 27\% (actualmente son el $34 \%$, aunque en números absolutos los niños seguirán siendo un importante contingente de alrededor de 10 millones de habitantes; los adultos mayores en ese año, representaran el $8 \%$, actualmente son el $5 \%$ ).

Este cambio de proporciones situará al Perú entre los países de "envejecimiento incipiente".

A continuación expondremos algunas cifras demográficas importantes, tales como:

Población del año 2002, fue de 26.7 millones de habitantes.

Población del año 2003, fue de 27.148 millones de habitantes.

Población proyectada al 2006, fue de 28.044 millones de habitantes.

El Censo poblacional del 2005, dio una cifra de 27 millones de habitantes.

Población proyectada al año 2010, será de 30 millones de habitantes.

Población proyectada al año 2020, será de 34 millones de habitantes.

Población de menores de 15 años al 2003, fue del 33\% de la población.

El Perú representa 11 eco-regiones naturales.

El Perú representa 3 regiones naturales.

La costa alberga al $52 \%$ de la población.

La sierra alberga al 34\% de la población. 
La selva alberga al $14 \%$ de la población.

La ciudad de Lima Metropolitana alberga al 30\% de la población.

En el año del 2001 trabajaban 1.219, 000 niños.

Del total de la población de 6 a 17 años de edad, estimada en 6.943,000 habitantes, se encontraba ocupada cerca del $29 \%$, de ella cerca del $80 \%$ asiste a los centros educativos, sin embargo el $68 \%$ es analfabeta.

Los niños entre 6 a 16 años de edad, que suman 1, 253, 000 habitantes, no concurren a las escuelas.

El 54\% de la población peruana es pobre, siendo los más afectados los niños.

La pobreza no extrema en el 2002, era el 39\% de la población.

La pobreza extrema era el 15\% de la población total, en el año 2002.

La desnutrición crónica, compromete al $25.4 \%$ de niños menores de 5 años.

La incapacidad de tener recursos financieros suficientes aflige al $70 \%$ de la población peruana.

En Lima Metropolitana, considerada la mejor zona socioeconómica del país, los pobres son el 44\%, (los pobres no extremos el 39\% y los extremos son el $5 \%$ ).

El Perú, es un país, donde la población menor de 15 años de edad, es del 33\% y dentro de ésta, la población pre-escolar (hasta antes de cumplir los 6 años de edad), ocupa un lugar muy importante, con el 14.25\% según el INEI 1993, de dicha población; la cual configura que estas edades, sean ampliamente mayoritarias, es decir; nuestro país está poblado ahora por gente de muy corta edad, pero paradójicamente, es un grupo poblacional al cual se le presta muy poca o casi ninguna atención, por decir lo menos; desde la perspectiva de la salud mental, pues se desconoce con propiedad la prevalencia actual de los múltiples trastornos psicopatológicos y psiquiátricos que puedan afectarla, desde el punto de vista de las edades que comprende, así como las características de estas entidades clínicas que también puedan afectarla y cuáles son los estratos socio-económicos significativamente más comprometidos; pues los datos que se conocen y se usan, generalmente, están basados en estudios y referencias extranjeras, las cuales como es lógico de suponer, distan mucho de aproximarse al conocimiento de lo que acontece en nuestra realidad; además los hallazgos locales tienen la dificultad de no poder extrapolarse, por la heterogeneidad poblacional, debido a las características étnicas, sociales, educacionales, culturales, económicas y geográficas de nuestro país.

Así, tenemos por ejemplo, que las referencias y cifras de prevalencia actual, en cuanto a ciertos comportamientos anormales, trastornos psicopatológicos o clínicos, tales como la epilepsia, el retraso mental, la enuresis, la encopresis, los tic, las dificultades en el habla, los trastornos de conducta, los problemas del aprendizaje social inicial, los trastornos del desarrollo, los trastornos afectivos, y aquellos trastornos que tienen que ver con los procesos de ansiedad, entre los que se pueden mencionar a la onicofagia, la succión del dedo pulgar, el temor a la oscuridad, el temor a separarse de la figura materna, la dificultad de adaptación a la escuela inicial, la hiperactividad motora, la masturbación compulsiva, 
la mentira patológica, el hurto; entre algunos; sin olvidar aquellos trastornos psicopatológicos que se dan tempranamente en edad, los cuales con el paso del tiempo habrán de predisponer y dar en última instancia los trastornos de personalidad, las reacciones o trastornos neuróticos, las manifestaciones psicopáticas o antisociales, los trastornos orgánico cerebrales, y los trastornos psicóticos, en todos los cuales, como lo refieren los teóricos y estudiosos de la Psicología Infantil, la Psiquiatría Infantil, la Psicopatología del Desarrollo y la experiencia clínica en el quehacer directo con los niños, se inician mayormente en la edad pre-escolar. Es por todo ello que los estudios epidemiológicos que nos aproximen, así sea de una manera muy discreta a esta realidad, serán de un gran valor, al inicio, en una labor en el campo de la Prevención Primaria, al conocer la prevalencia actual y posteriormente, con el conocimiento cuántico de cada una de estas entidades, constituirse estos hallazgos en el referente y elemento auxiliar indispensable y fundamental para los trabajadores de la Salud Mental Infantil y Disciplinas Afines.

Volvemos a señalar que los estudios epidemiológicos en la edad pre-escolar, en nuestro medio son casi inexistentes, los pocos estudios, de los cuales se pueden obtener datos epidemiológicos de prevalencia actual, se dan en poblaciones de riesgo donde la prevalencia actual, de manera general o global, se considera, puede situarse entre el $35 \%$ y el $45 \%$, pero las cifras de prevalencia actual, que nosotros vamos hallando, desde el primer estudio del año 2000 hasta el año 2010, se encuentran en porcentajes mucho más elevados, evidenciándose de esta manera la magnitud y la importancia del problema a estudiar, razón por la cual cada año, en cada nueva investigación, si bien es cierto, abordado el tema epidemiológico, este va haciéndose progresivamente más selectivo, y ahora en el año 2013, nuestro estudio va dirigido a la compilación, contrastación, condensación, comparación y fusión de los hallazgos encontrados en las investigaciones precedentes en esa línea, suponiendo, como es lógico, en una aproximación discreta, pero no por ello menos importante y valiosa.

En los años del 2000, 2001, 2002, 2003 utiliza el instrumento llamado IPRESCO, En el 2004, Oliver, siguiendo la misma línea de investigación de los años precedentes, así como en un universo de estudio muy similar, pero esta vez abreviando su instrumento Ilamándolo IPRESCO-ABREVIADO o (IPRESCO-A).

En el año del 2005, utiliza como base el instrumento del año precedente IPRESCOABREVIADO (IPRESCO-A); pero esta vez abreviado y revisado, al que denomina IPRESCO-ABREVISA.

En el estudio del 2006, el investigador hace uso de un nuevo instrumento basado e inspirado en todos los anteriores instrumentos aplicados, pero al cual denomina IPSIN, y tiene sus connotaciones muy propias y particulares, en sus hallazgos de prevalencia actual, presenta cifras ya más exactas, el de un $75 \%$ de morbilidad, y lo que realmente acontece en los estratos representativos de la población de niños limeños menores de seis años de edad, pues el instrumento ya mejor afinado, de igual manera que la aplicación del mismo, con un número menor de encuestadores, la mejor preparación de éstos, conjuntamente 
con la seriedad del trabajo realizado, en virtud del profesionalismo; evitando tener muchas variables no controlables como parece haber sucedido en los estudios anteriores, servirán para corroborar los estudios anteriores y/o planear un estudio más extenso, en el sentido de abarcar un universo mayor y con un instrumento, si cabe el término más funcional, como el que se pretende presentar ahora, intentando hacerlo más flexible, fluido, menos complicado, el cual nos dé respuestas más precisas.

Así es importante el reflexionar en referencia que a pesar de las consecuencias de los comportamientos anormales, trastornos psicopatológicos y hasta psiquiátricos que trae, la ausencia de programas de intervención temprana, como la Prevención Primaria, hace que en lugar de disminuir estos procesos vayan en aumento; otras explicaciones para este incremento (referido al caso de los no pre-escolares específicamente), es el aumento en la expectativa de vida, la intensificación del estrés, la crisis de la familia y la falta de soporte social, la facilidad con la cual la oferta de las sustancias psicoactivas llegan a ellos, la vagancia, el pillaje en grupo, y en esencia la carencia de referentes de valor ético a los cuales recurrir o imitar, debido a la descomposición social de las últimas décadas, en las cuales las figuras públicas han caído, así como la descontrolada información deformada de la prensa en todas sus formas, al uso indiscriminado del Internet, que hacen que los niños en los hogares se vuelvan contestatarios y entiendan de manera equivocada su derecho a la libertad de opinión, probablemente por la globalización que los niños ahora también viven e influye sobre ellos, en edades en las cuales tienen que ser conducidos para estructurar una conducta acorde a los moldes, costumbres, valores e ideales adecuados de nuestra sociedad.

Pero todo lo mencionado no es sólo responsabilidad del niño sino de los progenitores, quienes también, por la lucha por la vida o subsistencia, abandonan muchas responsabilidades básicas, y dejan al niño sin control.

Según la dimensión actual del problema y de las proyecciones, no existen recursos económicos suficientes para atender esta gran demanda de Salud Pública, se requiere entonces del conocimiento detallado y minucioso de la epidemiología que nos conduzca a una programación racional de recursos, los cuales puedan cubrir la necesidad actual y prevenir el incremento en la demanda de los Servicios de Salud Mental, por el aumento directo y desmesurado de estos comportamientos anormales o procesos psicopatológicos, los cuales serán más evidentes y limitantes de una mejor calidad de vida, conforme las edades vayan siendo mayores, y los procesos mórbidos no pasen, como en la mayoría de casos, desapercibidos, en razón de la edad tan temprana del grupo menor de seis años de edad, sobre todo.

\section{Importancia de la investigación:}

La epidemiología es una disciplina de primera importancia, sobre todo en aquellos países donde hay una preocupación por hacer un afronte serio de la Salud en general, y en el 
campo de la Salud Mental, su importancia cobra una mayor relevancia en virtud de los grados de incapacidad, cronicidad y gasto económico que generan al Estado, a la Sociedad y a la Familia estos trastornos psíquicos.

En nuestro medio, siendo un país pobre y sin recursos para realizar estudios gubernamentales en el campo de la Salud, mucho menos interesa el campo de la Salud Mental, y dentro de ella, muy alejada de los intereses de los gobiernos de turno se halla el de la Salud Mental Infantil, la cual sigue siendo la gran postergada, no solamente por los organismos estatales y privados, sino por las investigaciones académicas, tal como lo demuestran la poquísima o carencia de tesis universitarias, artículos de investigación o de reflexión, abordaje en la formación de los futuros trabajadores de las Ciencias de la Conducta Humana y Disciplinas Conexas y en síntesis por la falta de interés de los profesionales relacionados con el trabajo infantil.

Los hallazgos a obtener siempre serán de mucho valor para las diversas Disciplinas Conexas con la Salud Mental en general y la Salud Mental Infantil en particular, tales como la Psicología Clínica, la Psicología Educacional, la Psicología Clínica y de la Salud, la Psiquiatría Infantil, la Psicopatología Infantil, la Psicopatología del Desarrollo, la Pediatría, la Neurología Infantil, la Educación Inicial, la Educación Primaria, la Psicoterapia Infantil, la Psicoterapia de Familia, y también para las ramas de la Economía y la Política, pues debido a la cronicidad y deterioro que muchas de estas entidades clínicas traen consigo, interesan no tan sólo desde una perspectiva médica, educativa, sino familiar, socio-económica y política.

La política general es la suma de las decisiones que hacen que la sociedad se configure de determinada manera. Proporciona un marco en el cual se desarrollan, por ejemplo, la producción industrial y agrícola, la gestión de las empresas y los servicios de salud. Perfila el abanico de opciones en el que las organizaciones y los individuos hacen sus elecciones y, por lo tanto, influye directamente sobre el ambiente y sobre los hábitos de vida.

La política sanitaria suele referirse específicamente a los aspectos de asistencia médica, pero la salud depende de una gran variedad de decisiones políticas que no se limitan al campo médico o sanitario. Por tanto, una verdadera política sanitaria proporciona un marco de las actividades de promoción de la salud, tanto en la economía general de una comunidad, como en la agricultura, la industria, el trabajo, la energía, el trasporte y la educación.

Si se quiere que la epidemiología tenga éxito en la prevención y en el control de las enfermedades, la investigación epidemiológica deberá influir sobre la política general y no sólo sobre la política sanitaria. Por el momento, la epidemiología no ha alcanzado, en nuestro medio, todas sus posibilidades a este respecto y son pocas las áreas en las que la investigación epidemiológica ha sido aplicada por completo. No obstante, cada vez es mayor el reconocimiento de la importancia de la epidemiología en la adopción de políticas concretas.

La influencia de la epidemiología suele estar mediada por la opinión pública.

Los políticos de muchos países, en lugar de dirigir la opinión pública, suelen ir detrás de ella; la atención que los medios de comunicación dedican a la investigación epidemio- 
lógica aumenta cada día y ha hecho que la opinión pública se sensibilice, la epidemiología influye muchas veces en la política general de manera relevante aunque casi siempre se tienen también en cuenta otros factores.

En 1986, la Carta de Ottawa para el Fomento de la Salud, estableció claramente que en la salud influyen decisiones políticas en muy diversos campos.

La política sanitaria no es responsabilidad única de los ministerios de salud. Las políticas de muy diversas instituciones gubernamentales y no gubernamentales tienen repercusiones importantes sobre la salud.

En todos los campos de la política general debe haber una preocupación constante por la salud y la equidad. Las políticas agrícolas influyen sobre la disponibilidad, precio y calidad de sus productos; las políticas fiscales y de publicidad influyen sobre el precio y la disponibilidad de los productos; la política del transporte influye sobre la magnitud de la contaminación ambiental urbana y sobre el riesgo de los accidentes de tránsito.

En muchos países, la estrategia de salud para todos de la Organización Mundial de la Salud (OMS), proporciona una base para la política sanitaria. Un aspecto fundamental de esta estrategia es el establecimiento de objetivos y metas de salud. El enfoque varia, pero en todos los países esos objetivos se establecen tomando como base el conocimiento epidemiológico.

\section{Objetivo general}

Estará dirigido a cuantificar, los trastornos psicopatológicos de los niños menores de catorce (14) años de edad, de los diferentes estratos socioeconómicos de la ciudad de Lima Metropolitana, que se han estudiado, señalando la prevalencia promedio actual, los trastornos psicopatológicos, la morbilidad correspondiente, en porcentajes y de manera general.

\section{Hipótesis}

Estas son:

1.- "Hay en los niños limeños menores de catorce años de edad, un porcentaje altamente significativo de trastornos psicopatológicos por áreas, que se deben precisar, comparándolos con anteriores estudios semejantes".

2.- "Hay en los niños limeños menores de catorce años de edad, una serie de trastornos psicopatológicos, los cuales hay la necesidad de precisarlos, comparándolos con los anteriores estudios semejantes".

3.- "Hay en los niños limeños menores de catorce años de edad, una serie de trastornos psicopatológicos, los cuales hay la necesidad de cuantificarlos de manera independiente, para luego realizar un estudio comparativo y correlacional, lo cual nos dará la incidencia". 
4.- "Hay en los niños limeños menores de catorce años de edad, una serie de indicadores de psicopatología, los cuales hay la necesidad de precisarlos, comparándolos y describiéndolos con los estudios similares, anteriormente realizados".

5.- "Hay en los niños limeños menores de catorce años de edad, una serie de indicadores de psicopatología, los cuales hay la necesidad de cuantificarlos, comparándolos, describiéndolos y correlacionándolos, con los estudios semejantes anteriormente realizados".

6.- "Hay la necesidad, basados en los estudios anteriores similares, el de poder sacar la incidencia de estas entidades psicopatológicas".

\section{Aspectos metodológicos de la investigación epidemiológica en psicopatología infantil}

\section{Método y procedimiento}

Si bien es cierto, para los investigadores en general, la metodología a utilizar en una investigación, no es motivo de mayor problema o preocupación; pero en el campo de la investigación en psicopatología infantil, el método se torna un poco más complicado, en razón de las condiciones socio-demográficas y patológicas de la calidad sui generis del universo a investigar.

Se tienen que considerar algunas reflexiones, en torno a lo que podemos denominar "Sistema Básico de Muestreo", para garantizar su representatividad en los diversos niveles socioeconómicos, dirigidos a nuestro estudio epidemiológico de prevalencia actual, de los indicadores específicos de los trastornos psicopatológicos, en los niños limeños menores de catorce años de edad.

Una parte importante de la investigación en Ciencias Humanas, en las Ciencias de la Conducta Humana, en el Área Clínica, en el Área de la Psicopatología Infantil; en la Psicopatología del Desarrollo, es la investigación basada en muestras de sujetos.

En el caso específico de los estudios epidemiológicos, las muestras adquieren una importancia mucho mayor, por cuanto se tienen que abordar los aspectos de ella y las variables a estudiar, planteándonos que ellas tienen que ser las que mejor controlemos, así como el factor del tiempo, por cuanto, la prevalencia actual, va a estar referida al hallazgo en un aquí y ahora del proceso mórbido.

Dos son los aspectos que definen la pertinencia de un sistema de muestreo apropiado para propósitos de adaptación: la aleatoriedad y la representatividad.

a. La aleatoriedad, la cual supone tener evaluados, seleccionados, bajo un sistema probabilístico, el cual garantiza una misma probabilidad para todos los sujetos inmersos en la investigación que se está desarrollando. 
b. La representatividad, se refiere más bien a garantizar la idoneidad de la muestra, para representar a la población, es decir, para parecerse mucho más a ella, de manera casi real, por ello el universo tiene que ser escrupulosamente seleccionado.

Tanto la aleatoriedad como la representatividad, buscan minimizar la presencia del sesgo, es decir, la posibilidad de error, o un error mayor en los resultados. Existen diversos tipos de sesgos que pueden producirse: ajenos o no al sistema de muestreo empleados. Pero evitar el sesgo supone tener un sistema de muestreo muy caro económicamente, al menos para los investigadores individuales de nuestro medio; un sistema de muestreo razonable no sólo es caro en su formulación, sino fundamentalmente en su ejecución, en su procedimiento, en su desarrollo. A ello hay que agregar que es difícil tener marcos de muestras apropiadas, pues lamentablemente se carece de tal información.

La aleatoriedad y la representatividad no son fáciles de obtener. Es común observar el error de muchos investigadores mal informados, que suponen que usar una fórmula de tamaño de muestra basado en un diseño probabilístico hace aleatorio el sistema de muestreo. Pero aún es más preocupante que su selección sea intencional, sin explicar la representatividad de ella, es decir, sin definir ni justificar a quien o a quienes representa realmente la muestra. Aunque es plausible sacrificar la aleatoriedad, de ninguna manera se puede sacrificar la representatividad. No se trata de decir, tengo una muestra, qué se puede hacer; se trata de definir una población y definirla en un sistema de muestreo que la represente. Mejor aún si la selección de unidades del muestreo se obtienen al azar.

Otro caso que ocurre con frecuencia en los estudios epidemiológicos, es trabajar con edades comprendidas en la escolaridad, bien sean estas la educación primaria, la educación secundaria o la educación superior o universitaria, que pese a desbordar la edad propiamente escolar, siempre es estudiada; pero nosotros vamos a estudiar también a niños menores de los seis años de edad, es decir, aquellos que corresponden a la edad pre-escolar y escolar primaria, y ahí radica otra nueva dificultad. Los sistemas de muestreo elementales suponen que es posible muestrear individuos a partir de un marco teórico. Pero como hemos expresado antes, los investigadores no disponemos de tal marco, pues ellos a lo sumo pueden obtener, con sacrificio un marco de muestras de los Centros de Salud, de Centros Educativos, de Hospitales, de los Clubes de Madres, de los Comedores Populares, etc., sean en el ámbito de un grupo de éstos, de un Distrito, o de un estrato socioeconómico determinado. Para evaluar en este rango de edad, hay que reconocer que los participantes se encuentran mayoritariamente en Cunas, en Centros pre-escolares y escolares primarios, algunos acuden a los Hospitales Generales, a los Centros de Salud para los programas de control del niño sano, otros acompañan a sus madres a los mercados de abastos o final y mayoritariamente se hallan en sus domicilios, todo ello dentro de las diversas jurisdicciones tipos Asentamientos Humanos, Centros Poblados Rurales, Barriadas, Invasiones, Centros Urbanos, etc.; de los Distritos correspondientes y de las diversas Provincias, que corresponden a los Departamentos del país y finalmente al propio país. 
Evaluar a participantes en el rango de 0 meses a 5 años con 11 meses de edad, de manera confiable, lo cual supone pasar de sistemas de muestreo elementales a sistemas de muestreo más complejos, por etapas, tomando en cuenta diversas jerarquías, hasta llegar al pre-escolar y escolar primario propiamente dicho. $Y$ de los 6 años de edad hasta los 13 años con 11 meses, plantea el mismo reto metodológico. Por otro lado, un tipo de muestreo de interés en los estudios epidemiológicos, es investigar por niveles socioeconómicos. Este problema no se encuentra resuelto en nuestro medio. Por ello, se dan las posibilidades de tomar el nivel socioeconómico de la institución a la que pertenece el participante, la zona donde está su domicilio, a la condición de empleo de sus progenitores, u otras condiciones que nos permitan asignársela a él (el sujeto de investigación) la condición socio económica correspondiente, merced a indicadores directos o mayormente indirectos. En cada caso no es fácil encontrar una manera convincente de muestrear por nivel socioeconómico. En el primer caso, se tendría que evaluar el nivel socioeconómico de cada sujeto, es decir, el muestreo se define a posteriori. Además se requiere tener información sobre diversos aspectos para considerarlos en un indicador socioeconómico, y esto supondría una encuesta propia. Así también, los participantes no sólo tienen que ser confiables al dar ésta información, sino que deben de ser capaces de darla, cosa que puede ser más complicada en los niveles pre-escolares, aun tomando la encuesta a las madres.

En el otro caso, no se puede llegar al hecho simple de asignar el nivel socioeconómico a la institución (cuna, centro de salud, mercado, domicilio, etc.), y al individuo que se encuentra en ella. Se tiene que encontrar un sistema convincente de asignación de niveles socioeconómicos a las instituciones y con esto asignársela a los que concurren a ellas.

Los sistemas de muestreo más eficientes son aquellos que permiten establecer y manejar márgenes de error, y que además permiten realizar estimaciones de errores de las muestras. Estos muestreos son probabilísticos; pero si éstos no son posibles de realizar, por algunas de las razones que hemos expuesto, los muestreos no probabilísticos por etapas que garantizan representatividad y proporcionalidad pueden ser una buena alternativa. En todas las investigaciones, se han tratado de presentar un sistema básico de muestreo para garantizar representatividad por niveles socioeconómicos, en Distritos-tipo de Lima Metropolitana, para el estudio de la prevalencia actual epidemiológica de los trastornos psicopatológicos en niños menores de catorce años de edad.

Este sistema de asignación sólo permitirá trabajar los niveles socioeconómicos entre instituciones pero no dentro de ellas. Si se quiere llegar a ese detalle, que no es de interés, del presente estudio epidemiológico, se tendría que buscar un indicador común para todos los sujetos.

Creemos que en este sistema de muestreo que se presenta puede tenerse una alternativa razonable para realizar el muestreo en los estudios epidemiológicos. Esta alternativa no pretende sustituir a sistemas más efectivos, pretende sustituir a sistemas más bien erróneos que han predominado en la investigación epidemiológica infantil en nuestro país. 


\section{Metodología}

El método empleado en las investigaciones fueron múltiples, así:

a. Descriptivo; por cuanto se describirán los trastornos psicopatológicos y los indicadores correspondientes que se hallen.

b. Exploratorio; se indagará tratando de encontrar los indicadores específicos de los trastornos psicopatológicos.

c. Observacional; centrada en la percepción directa del encuestador y de lo informado por la madre y por el niño cuando así lo amerite.

d. Transversal; los estudios se situaron en un tiempo determinado, haciendo un corte vertical en ese tiempo, el cual fue el momento del estudio.

e. Comparativo; se tendrá como referentes a estudios similares, en su totalidad o parcialmente, realizados con anterioridad y que tengan alguna conexión con el que se está desarrollando, así como cotejarlas entre ellas, motivo del presente estudio.

f. Clínico; por cuanto se buscarán los indicadores de los trastornos psicopatológicos, los cuales en esencia son netamente clínicos.

g. Correlacional; por cuanto se buscaran que unir, juntar, relacionar, los datos de las investigaciones anteriores, buscando coincidencias válidas.

\section{Población}

Se estudiará la población de todas las investigaciones anteriores de carácter semejante, es decir de niños menores de catorce años de edad de Lima Metropolitana. En investigaciones anteriores (Oliver, 2000, 2001, 2002, 2003, 2004 y 2005, 2006a, 2006b, 2007, 2008, 2009, 2010), encontró que el $15 \%$ de las madres con estas características fueron evaluadas en los Centros de Salud, un 65\% fueron evaluados en los Centros Pre-escolares, un 10\% en los Mercados como acompañantes de sus madres y finalmente el otro $10 \%$ en sus domicilios, sobre todo en las investigaciones que comprendían a los niños en edades pre-escolares; en los niños escolares, en las escuelas y lugares donde podían haber de esa edad.

Según el INEI (1999); la distribución de la población por niveles socioeconómicos a fines de 1998 fue de:

a. El 6.21\% para el nivel socioeconómico alto, o clase A.

b. El $16.73 \%$ para el nivel socioeconómico medio alto, o clase $B$.

c. El $22.08 \%$ para el nivel socioeconómico medio, o clase C.

d. El 21.55\% para el nivel socioeconómico medio bajo, o clase D.

e. El $33.43 \%$ para el nivel socio económico bajo, o clase E. 


\section{Tamaño de la muestra}

Como se requiere la representación de los cinco niveles socioeconómicos y de las tres instituciones, habrán de tenerse 15 combinaciones posibles para representar. En cada combinación se espera como mínimo un promedio de 135 a 150 madres que respondan el instrumento. Para lograr esto se consideró un tamaño de muestra de 2500 a 3000 sujetos, para garantizar todas las posibles comparaciones deseadas, en la mayoría de los estudios en pre-escolares.

En las edades escolares se le tuvo como referencia, pues aquí se aplicó el instrumento en las escuelas y lugares donde se hallaran estos niños.

Si tenemos en consideración los últimos datos poblacionales del INEI (2009), que la población total del país es de 30 millones de habitantes, de los cuales el 14.25\% es menor de 6 años de edad, es decir, 4, 275,500 habitantes; la población de Lima Metropolitana que alberga al 30\% de la población nacional, con 9,000,000 de habitantes, correspondiendo $1,282,500$ a los menores de 6 años de edad con el 14.25\%. Menores de 14 años serán el 38\%, siendo 11, 400, 000 habitantes; y en Lima Metropolitana serán 3, 420, 000 habitantes. Es decir tenemos un marco poblacional de aproximadamente $4,600,000$ habitantes menores de catorce años.

Este es el marco numérico al que hay que tener presente y referirse para darse cuenta de la magnitud del problema a estudiar, a nivel cuántico, para un mejor y cuidadoso abordaje, que nos de cómo resultados, cifras más cercanas a la realidad que pretendemos investigar.

\section{Procedimiento}

Para garantizar la representatividad de la muestra respecto a la población, el muestreo será en varias etapas, es decir, polietapico, porque comprenderá las siguientes dos etapas sucesivas que se reconocen para llegar a los sujetos de investigación, donde se tiene que pasar por lo menos dos etapas obligatoriamente.

En este caso, en una primera etapa se seleccionarán los Distritos por sus estratos socioeconómicos y en una segunda etapa a las Instituciones.

\section{Selección de los distritos de Lima Metropolitana}

En base al plano de Manzanas del INEI (2003), los distritos se han ubicado en un estrato socioeconómico (Alto o A, Medio Alto o B, Medio o C, Medio Bajo o D y Bajo o E.), sobre la base del porcentaje poblacional mayoritario existente según dicho estudio.

De un total de 43 distritos de Lima Metropolitana, se seleccionó el 25\%, el porcentaje puede ser otro pero éste es el más común, es decir aproximadamente once (11) distritos para luego determinar en ellos la muestra de las instituciones. 
Esto significa que habrá más de un distrito, en algunos niveles socioeconómicos para los once (11) distritos de la lista, completando los distritos distribuidos proporcionalmente, en los estratos con más de nueve (9) distritos.

Según el cuadro anterior se tendrá:

2 distritos del nivel Alto o $\mathrm{A}$;

2 distritos del nivel Medio Alto o $B$;

1 distrito del nivel Medio o $\mathrm{C} ; \mathrm{y}$

6 distritos del nivel Bajo, distribuidos en 4 distritos del nivel Medio Bajo o Dy 2 distritos del nivel Bajo o E, o más.

Así seleccionados los distritos, se tomarán 2 distritos del nivel socioeconómico Alto, y para el caso del nivel socioeconómico Medio Bajo se tomará 4 distritos ya seleccionados del nivel socioeconómico Bajo para tener los 11 distritos considerados a ser investigados.

En base al plano de Manzanas del INEI (1999), los distritos se han ubicado en un estrato socioeconómico (Alto, Medio Alto, Medio, Medio Bajo y Bajo), sobre la base del porcentaje poblacional mayoritario existente según dicho estudio.

De un total de 43 distritos de Lima Metropolitana, se seleccionará el 15\%, el porcentaje puede ser otro pero éste es el más común, es decir aproximadamente siete distritos para luego determinar en ellos la muestra de las instituciones.

Esto significa un distrito dentro de cada nivel socioeconómico para cada seis distritos de la lista, completando los distritos distribuidos proporcionalmente, en los estratos con más de seis distritos.

Según lo presentado se tendrá:

1 distrito Alto;

1 distrito Medio Alto,

1 Distrito Medio,

4 Distritos Bajos, distribuidos en 2 distritos Medio Bajos y 2 distritos Bajos.

Aunque el número es menor, se tomará un distrito del nivel socioeconómico Alto, y para el caso del Nivel socioeconómico Medio Bajo se tomará otro distrito ya seleccionado del nivel socioeconómico Bajo para tener los siete distritos considerados a ser investigados.

Los Distritos seleccionados en los estudios fueron los siguientes: Año 2000:

Bajo: San Juan de Miraflores.

Villa María del Triunfo.

Villa El Salvador.

Año 2001:

Alto: Miraflores.

Medio Alto: Santiago de Surco.

Medio: San Martín de Porres.

Medio Bajo: Chorrillos y Los Olivos.

Bajo: Santa Anita y San Juan de Luringancho. 
Año 2002:

Alto: Miraflores.

Medio Alto: Santiago de Surco.

Medio: San Martín de Porres.

Medio Bajo: Chorrillos y Los Olivos.

Bajo: Santa Anita y San Juan de Luringancho.

Año 2003:

Alto: Miraflores.

Medio Alto: Santiago de Surco.

Medio: San Martín de Porres.

Medio Bajo: Chorrillos y Los Olivos.

Bajo: Santa Anita y San Juan de Luringancho.

Año 2004:

Alto: San Isidro.

Medio Alto: Santiago de Surco.

Medio: San Luís.

Medio Bajo: Comas y Ate-Vitarte.

Bajo: Pachacamac y Lurín.

Año 2005:

Alto: Miraflores.

Medio Alto: La Molina.

Medio: San Martín de Porres.

Medio Bajo: Chaclacayo y Chorrillos. (Zonas centrales).

Bajo: Carabayllo y El Agustino. (Zonas periféricas).

Año 2006:

Alto: Miraflores.

Medio Alto: San Miguel.

Medio: San Martín de Porres.

Medio Bajo: Comas y San Juan de Lurigancho (Zonas Centrales).

Bajo: Villa María del Triunfo. San Juan de Miraflores.(ZP).

Año 2007:

Alto: Miraflores.

Medio Alto: San Miguel.

Medio: San Martín de Porres.

Medio Bajo: Comas. San Juan de Lurigancho. (Zonas Centrales)

Bajo: Comas. San Juan de Miraflores. (Zonas Periféricas).

Año 2008:

Alto: San Isidro.

Medio Alto: Jesús María.

Medio: San Luis. Surquillo. Rímac. 
Medio Bajo: Comas. San Juan de Lurigancho (Zonas Centrales).

Bajo: Comas. San Juan de Miraflores. (Zonas Periféricas). Año 2009:

Alto: Miraflores.

Medio Alto: San Miguel.

Medio: San Martín de Porres.

Medio Bajo: Comas y San Juan de Lurigancho.

Bajo: Villa María del Triunfo. San Juan de Miraflores. Año 2010:

Se seleccionaron 35 distritos, siendo los siguientes: Ancón. Ate-Vitarte; Barranco. Breña. Cieneguilla. Comas. Chaclacayo. Chorrillos. El Agustino. Jesús María. Independencia. La Molina. La Victoria. Lima-Cercado. Lince. Los Olivos. Lurín. Magdalena. Miraflores. Pachacamac. Pueblo Libre. Puente Piedra. Rímac. San Borja. San Isidro. San Juan de Lurigancho. San Juan de Miraflores. San Luis. San Martin de Porres. San Miguel. Santa Anita. Surco. Surquillo. Villa El Salvador y Villa María del Triunfo.

\section{Selección de las instituciones}

Para la selección de las instituciones, en las 15 combinaciones posibles, fue necesario diseñar una estrategia de trabajo de campo, que garantice la idoneidad de éstas para representar el nivel socioeconómico correspondiente. De acuerdo al tamaño prefijado de las madres por instituciones y niveles socioeconómicos de la etapa anterior, se aconseja una selección, considerando las características visibles de estas, pero tomando en cuenta las preguntas socio-demográficas, que se incorporan en la encuesta. Adicionalmente se usara la dirección de la institución, o del domicilio para encontrar la asignación del nivel socioeconómico, dado en el Plano de Manzanas del INEI (2003), el cual será una referencia básica, la cual, luego se cotejará con las características ectoscopicas de la vestimenta, la salubridad de las zonas, la salubridad de las cunas, la nutrición del niño, las características externas e internas de la vivienda del niño y el grado de instrucción de las madres, sólo como referentes socioculturales y económicos.

En el caso de los escolares primarios, se les contactara en sus respectivas escuelas y en aquellos lugares de concurrencia más frecuente.

\section{Definición de la muestra}

La selección de la muestra es no probabilística, ya que se realizará de manera intencional, proporcional al tamaño; de acuerdo a dos criterios de estratificación.

Estrato socioeconómico INEI (2003), e instituciones donde se encuentran a los niños pre-escolares, Oliver (2000, 2001,2002, 2003, 2004 y 2005, y 2006a , 2006b, 2007, 2008, 2009 
y 2010 en niños escolares primarios; así como en dos etapas, es decir, bíetapica porque se seleccionaran distritos en una primera etapa e instituciones en la segunda etapa.

En lo que respecta al género, no se consideró como criterio de estratificación, pero de busco que la muestra fuese lo más uniforme posible, igual sucedió con las edades de los pre-escolares, en sus diversas subdivisiones, tratando de que fuesen, también, lo más homogéneos posibles, y para ambos fines se tomaron mayores encuestas que las obligatorias, con la finalidad de anular al azar, las sobrantes, para equiparar el sexo y las edades correspondientes. Igual consideración se tomó para los niños escolares primarios.

\section{Dominios de la muestra}

Para efectos del estudio se considerarán los siguientes criterios:

Criterios de inclusión

a. Madres de niños pre-escolares que acceden a la encuesta. Niños escolares primarios.

b. Instituciones clasificadas en diferentes niveles socioeconómicos.

La clasificación se obtendrá según la infraestructura y su ubicación en el Plano de Manzanas de Lima Metropolitana, según niveles socioeconómicos del INEI (2003).

c. Edad de las madres, mayores de los 18 años y menores de los 36 años de edad, para los estudios de niños pre-escolares.

Criterios de exclusión:

a. Relacionada con las características de la muestra:

Fuera del rango de edad del niño pre-escolar y escolar.

Falta de datos, sobre todo los socio demográficos.

b. Relacionada con la respuesta:

Respuesta excesiva: cuestionarios con más de cuatro preguntas contestadas positivamente.

Respuesta azarosa: cuestionarios que evidencian patrones sistemáticos de respuestas azarosas, o que se entregan en un tiempo extremadamente breve, o se evidencia desgano o falta de interés al responder.

Estas respuestas denotan poca colaboración del o la participante.

c. Pruebas que según protocolo sean inválidas.

Sobre respuestas: cuestionarios en los cuales se hayan contestado masivamente, bien sea con un No o un Sí.

\section{Procedimiento}

\section{Técnica de recolección de datos}

Una vez que se diseñaron los instrumentos específicos, a los cuales se les denomino de diferentes maneras, en el tiempo, pero todos los cuales servían de base para la caracteri- 
zación de los comportamientos anormales en niños limeños menores de catorce años de edad, se consideró necesario el precisar la muestra en los sujetos por cada distrito elegido, teniendo en consideración el estrato socioeconómico.

Para la recolección de datos se aplicó, en el trabajo de campo el instrumento respectivo, a las madres de los niños menores de 6 años de edad, elegidos al azar, que concurriesen al control del niño sano, en los hospitales, centros de salud, postas médicas, algunos consultorios médicos y psicológicos privados, en las guarderías, en las zonas de concentración de madres de familia como los mercados, clubes de madres y en la visita casa por casa; las madres podían contestar más de un instrumento, si tenían otros hijos menores de los 6 años de edad y a los niños en edad escolar, en las escuelas respectivas de la zona.

Las madres podían marcar varias preguntas, si el niño las tenía, lo cual era muy difícil, pues el instrumento, sólo podía permitir como máximo hasta tres respuestas, si había más de ese número se desechaba el instrumento.

En el caso de los niños en edad escolar, se les tomo en sus centros escolares educativos, de los salones escogidos al azar y por alumnos impares de la lista, así como en otros lugares donde hubiere niños de estas edades, es decir, parques, mercados, clubes vecinales, etc.

\section{Técnica de procesamiento y análisis de los datos}

Los datos obtenidos, se procesaron y analizaron de manera minuciosa y se catalogaron según el probable indicador del trastorno psicopatológico, en su área general correspondiente.

\section{Instrumentos}

Los instrumentos pasaron por distintas mutaciones, de acuerdo a las necesidades de las investigaciones, así se reajusto y abrevio el Inventario inicialmente usado en la primera investigación denominado IPRESCO, que tenía 147 preguntas, y fue aplicado en las anteriores investigaciones.

Para dar el IPRESCO-ABREVIADO (IPRESCO-A) con 109 preguntas, posteriormente, se mantuvo el número de preguntas, pero se cambió casi la totalidad de su forma de enunciado de las preguntas usando un lenguaje extremadamente sencillo, que estuviese al alcance de la comprensión de las madres. A este instrumento se le denomino IPRESCOABREVIADO Y REVISADO, (IPRESCO-ABREVISA), donde están considerados los principales trastornos psicopatológicos que se presentan en los niños menores de 6 años de edad, haciéndose un claro distingo entre ellos, de acuerdo a las edades de presentación y al desarrollo evolutivo del infante; donde se presentan dos opciones de respuestas, bien sea un "Si" o un "No".

Luego se elaboró un instrumento al cual se le denominó: "inventario de indicadores de trastornos psicopatológicos, en niños en edad escolar primaria, de 6 a 14 años de edad 
(EOP8)", conformado por 50 preguntas. El cual tenía un lenguaje sencillo para poder facilitar la comprensión de los niños de los 6 años de edad hasta los 14 años de edad.

En este instrumento están considerados los principales trastornos psicopatológicos que se presentan en los niños de 6 años de edad hasta los 14 años de edad, haciéndose un claro distingo entre ellos, de acuerdo a las edades de presentación y al desarrollo evolutivo del niño; donde se presentan dos opciones de respuestas, bien sea un "Sí" o un "No".

El instrumento EOP8, era el resultado de modificaciones de los instrumentos EOP6 y EOP7, usados en las investigaciones de los años del 2006 y 2007, modificaciones que permitieron hacer a EOP8 más versátil y funcional.

Dichos instrumentos pasaron previamente por un criterio de jueces, siendo estos pedíatras, psiquíatras infantiles, psicólogos infantiles, psicólogos educacionales, profesores de educación primaria de escuelas estatales y particulares y profesores universitarios del área, en número de 35, de los cuales se tomó en cuenta sus sugerencias, para el perfeccionamiento del cuestionario originalmente diseñado.

Sistematizándose en dos grandes áreas:

A. Área de las edades, la cual se distribuyó de acuerdo al desarrollo evolutivo, en los siguientes grupos de edades:

I. Del nacimiento al primer mes de edad.

II. De los 2 meses a los 6 meses de edad.

III. De los 7 meses a los 15 meses de edad.

IV. De los 16 meses a los 3 años de edad.

V. De los 3 años 1 mes a los 5 años con 11 meses de edad.

VI. De los 6 años hasta los 7 años con 11 meses de edad.

VII. De los 8 años hasta los 9 años con 11 meses de edad.

VIII. De los 10 años hasta los 11 años con 11 meses de edad.

X. De los 12 años hasta los 14 años de edad.

B. Área de los comportamientos anormales, que afectan o tienen que ver con la Salud Mental, en las siguientes áreas:

1. Trastornos del Sueño.

2. Trastornos de la Alimentación.

3. Trastornos de los Esfínteres.

4. Trastornos Motores.

5. Trastornos del Lenguaje.

6. Trastornos del Comportamiento.

7. Trastornos Afectivos.

8. Trastorno de la Sexualidad.

9. Trastornos Psicosomáticos.

10. Trastornos del Desarrollo. 


\section{Resultados más importantes de las investigaciones}

TABLA 1: SUJETOS MENORES DE 14 AÑOS DE EDAD INVESTIGADOS:

\begin{tabular}{lcc}
\hline AÑOS: & SUJETOS: & PORCENTAJES: \\
\hline 2000 & 600 & 3.41 \\
2001 & 1200 & 6.81 \\
2002 & 1000 & 5.68 \\
2003 & 1200 & 6.81 \\
2004 & 1200 & 6.81 \\
2005 & 901 & 5.12 \\
$2006^{\mathrm{a}}$ & 1510 & 8.57 \\
$2006^{\mathrm{b}}$ & 1800 & 10.22 \\
2007 & 1000 & 5.68 \\
2008 & 1500 & 8.52 \\
2009 & 3000 & 17.03 \\
2010 & 2700 & 15.33 \\
\hline TOTALES: & 17611 & 100.00 \\
\hline
\end{tabular}

Como se observa en la Tabla 1, en el año 2009 se tuvieron como sujetos de investigación al $17.03 \%$, del gran universo que se investigó, siendo el mayor cuantitativamente de los 12 investigados seguido del año 2010 con el 15.33 y si le añadimos el del 2006b con el 10.22, tendríamos un 42.58 del gran universo total, casi el 50\%, en solo 3 años.

GRÁFICO 1: SUJETOS MENORES DE 14 AÑOS DE EDAD INVESTIGADOS:

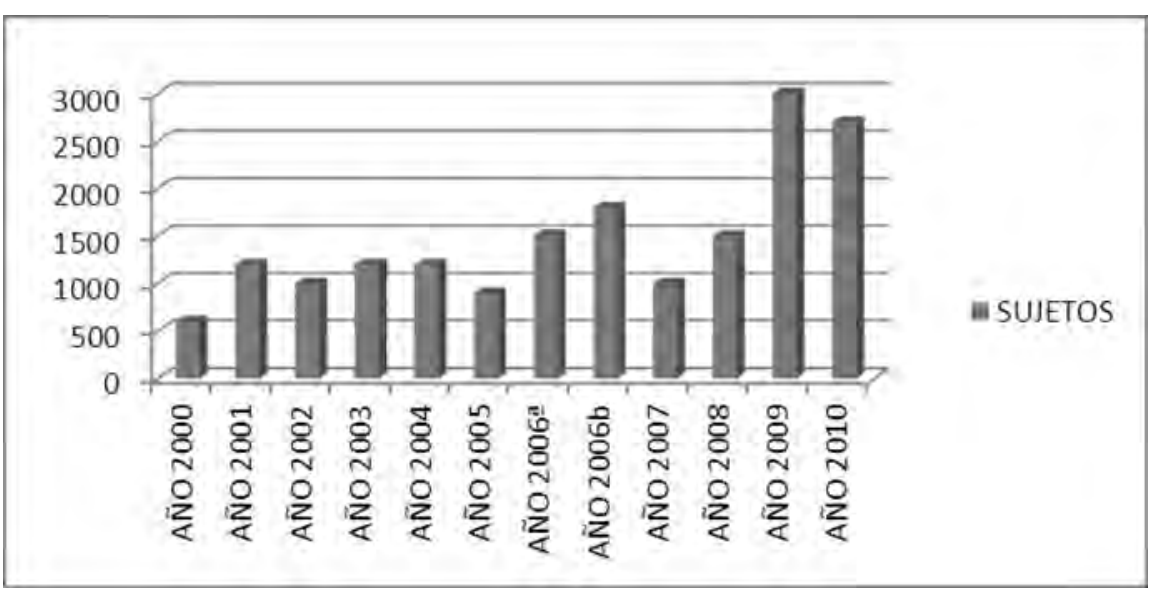


Como se observa en el Gráfico 1, en el año 2009 se tuvieron como sujetos de investigación al $17.03 \%$, del gran universo que se investigó, siendo el mayor cuantitativamente de los 12 investigados seguido del año 2010 con el 15.33\% y si le añadimos el del 2006b con el $10.22 \%$, tendríamos un $42.58 \%$ del gran universo total, casi el $50 \%$, en solo 3 años.

TABLA 2: PREVALENCIA HALLADA EN EL TIEMPO.

\begin{tabular}{cc}
\hline AÑOS: & PREVALENCIA: \\
\hline 2000 & 92.66 \\
2001 & 89.25 \\
2002 & 86.84 \\
2003 & 82.35 \\
2004 & 78.42 \\
2005 & 75.11 \\
$2006^{\mathrm{a}}$ & 67.08 \\
$2006^{\mathrm{b}}$ & 75 \\
2007 & 69.5 \\
2008 & 89.13 \\
2009 & 83 \\
\hline 2010 & 82.88 \\
\hline
\end{tabular}

Como se aprecia en la Tabla 2, la prevalencia más alta se obtuvo en la investigación del año 2000 con el $92.66 \%$ y la prevalencia menor en el año del $2006^{\mathrm{a}}$ con el $67.08 \%$ que evidencia un descenso progresivo, para en los años posteriores dar una curva de zigzag.

\section{GRÁFICO 2: PREVALENCIA HALLADA EN EL TIEMPO}

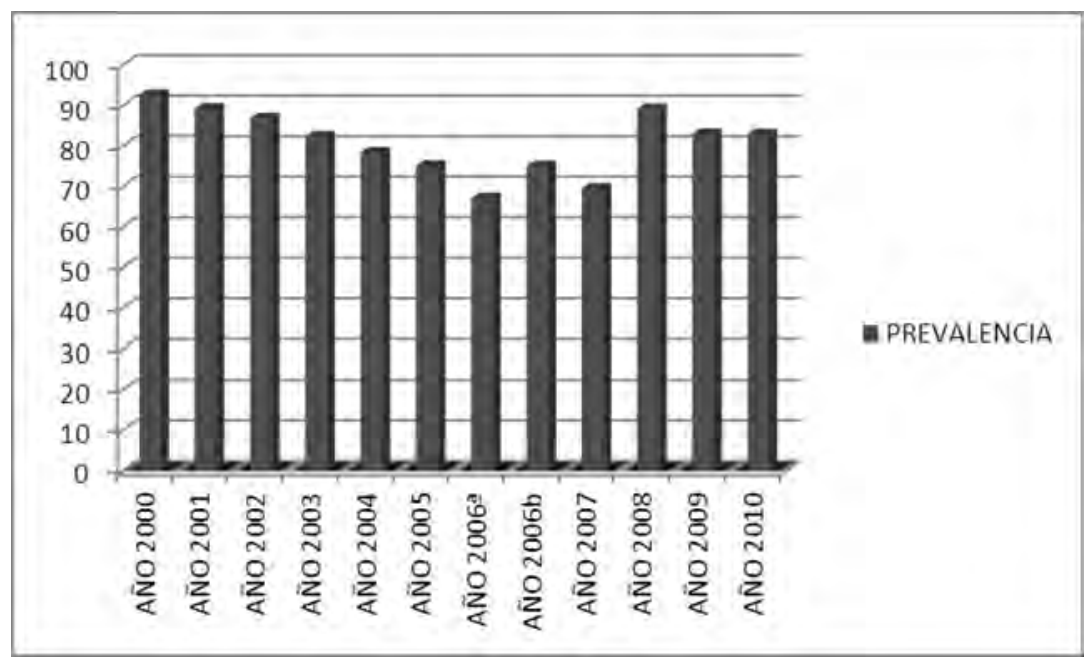


Como se aprecia en el Gráfico 2, la prevalencia más alta se obtuvo en la investigación del año 2000 con el $92.66 \%$ y la prevalencia menor en el año del $2006^{a}$ con el $67.08 \%$ que evidencia un descenso progresivo, para en los años posteriores dar una curva de zigzag.

TABLA 3: PROMEDIO DE LOS TRASTORNOS PSICOPATOLÓGICOS:

\begin{tabular}{lcc}
\hline TRASTORNO PSICOPATOLÓGICO & $\begin{array}{c}\text { GRAN TOTAL DEL TRASTORNO } \\
\text { PSICOPATOLÓGICO }\end{array}$ & $\begin{array}{c}\text { PROMEDIO DEL TRASTORNO } \\
\text { PSICOPATOLÓGICO }\end{array}$ \\
\hline COMPORTAMIENTO & 271.93 & 22.66 \\
DESARROLLO & 236.88 & 19.74 \\
AFECTIVIDAD & 226.8 & 18.9 \\
LENGUAJE & 108.22 & 9.02 \\
MOTRICIDAD & 81.41 & 6.78 \\
SUEÑO & 76.53 & 6.38 \\
ALIMENTACIÓN & 60.92 & 5.08 \\
PSICOSOMÁTICA & 54.9 & 4.57 \\
ESFÍNTERES & 38.18 & 3.47 \\
SEXUALIDAD & 37.34 & 3.4 \\
\hline
\end{tabular}

Como se ve en la Tabla 3, el mayor trastorno psicopatológico en su presentación es el referido a los trastornos del comportamiento con el $22.66 \%$, seguido de los trastornos del desarrollo con el $19.74 \%$ y los trastornos de la afectividad con el $18.9 \%$, sumando entre los tres el $61.3 \%$.

GRAFICO 3: PROMEDIO DE LOS TRASTORNOS PSICOPATOLÓGICOS:

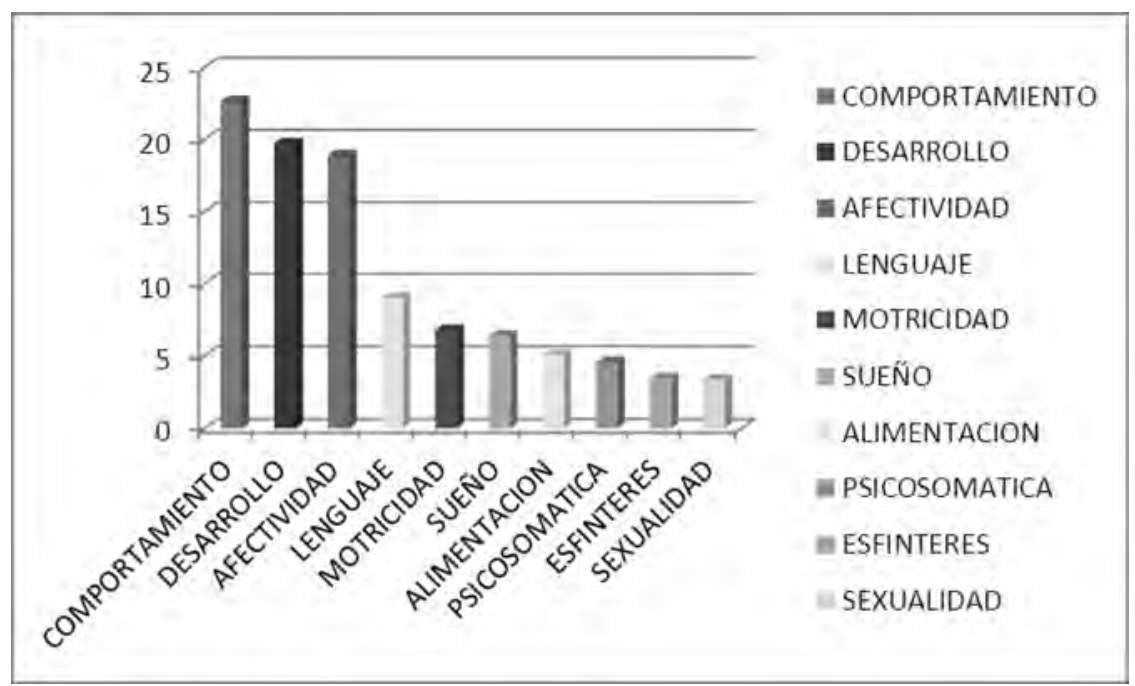


Como se ve en el Gráfico 3, el mayor trastorno psicopatológico en su presentación es el referido a los trastornos del comportamiento con el $22.66 \%$, seguido de los trastornos del desarrollo con el $19.74 \%$ y los trastornos de la afectividad con el $18.9 \%$, sumando entre los tres el $61.3 \%$.

TABLA 4: GRADOS DE ESTUDIOS.

\begin{tabular}{lll}
\hline PRE ESCOLARES: & & \\
\hline AÑO: & SUJETOS: & PORCENTAJES: \\
\hline 2000 & 600 & 3.4 \\
\hline 2001 & 1200 & 6.81 \\
\hline 2002 & 1000 & 5.68 \\
\hline 2003 & 1200 & 6.81 \\
\hline 2004 & 1200 & 6.81 \\
\hline 2005 & 901 & 5.12 \\
\hline 2010 & 1200 & 6.81 \\
\hline SUB TOTAL & 7301 & 41.44 \\
\hline ESCOLARES: & & \\
\hline AÑO: & SUJETOS: & PORCENTAJES: \\
\hline 2006 a & 1510 & 8.57 \\
\hline $2006 b$ & 1800 & 10.22 \\
\hline 2007 & 1000 & 5.68 \\
\hline 2008 & 1500 & 8.52 \\
\hline 2009 & 3000 & 17.05 \\
\hline 2010 & 1500 & 8.52 \\
\hline SUB TOTAL: & 10310 & 58.56 \\
\hline TOTALES: & 17611 & 100.00 \\
\hline
\end{tabular}

Como se ve en la Tabla 4, es superior con el $17.12 \%$ los sujetos escolares en relación a los pre-escolares de manera general; pero en los pre-escolares dentro de sí, guardan una relación casi igual, salvo el año 2000 que es cerca de la mitad menor. En los sujetos escolares todos los valores son casi similares salvo el año 2007 que difiere en menos, en casi cerca del $3 \%$. 
GRÁFICO 4: GRADOS DE ESTUDIOS

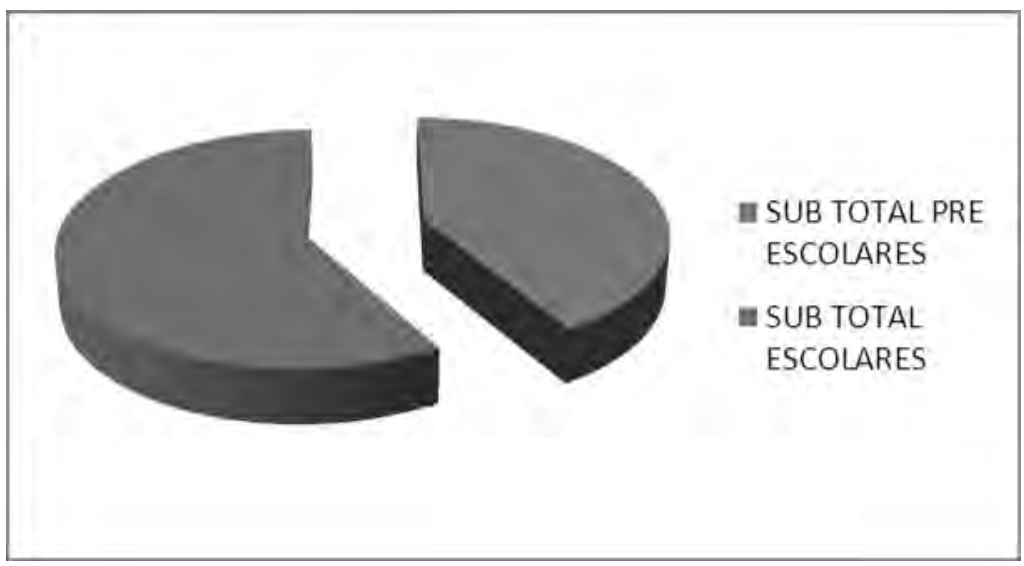

Como se ve en el Gráfico 4, es superior con el 17.12\% los sujetos escolares en relación a los pre-escolares de manera general; pero en los pre-escolares dentro de sí, guardan una relación casi igual, salvo el año 2000 que es cerca de la mitad menor. En los sujetos escolares todos los valores son casi similares salvo el año 2007 que difiere en menos, en casi cerca del 3\%.

TABLA 5: GÉNERO

\begin{tabular}{|c|c|c|}
\hline \multicolumn{3}{|c|}{ PRE ESCOLARES: } \\
\hline AÑO: & MASCULINO: & FEMENINO: \\
\hline 2000 & 300 & 300 \\
\hline 2001 & 635 & 565 \\
\hline 2002 & 500 & 500 \\
\hline 2003 & 598 & 602 \\
\hline 2004 & 598 & 602 \\
\hline 2005 & 523 & 378 \\
\hline 2010 & 599 & 601 \\
\hline SUB TOTAL: & $3753(51.40 \%)$ & $3548(48.60 \%)$ \\
\hline \multicolumn{3}{|l|}{ ESCOLARES: } \\
\hline AÑO: & MASCULINO: & FEMENINO: \\
\hline $2006^{a}$ & 755 & 755 \\
\hline $2006 \mathrm{~b}$ & 1293 & 507 \\
\hline 2007 & 500 & 500 \\
\hline 2008 & 829 & 671 \\
\hline 2009 & 1501 & 1499 \\
\hline 2010 & 768 & 732 \\
\hline SUB TOTAL: & $5646(54.76 \%)$ & $10310(45.24 \%)$ \\
\hline TOTALES: & 9399 (53.37\%) & $8212(46.63 \%)$ \\
\hline
\end{tabular}


Como se ve en la Tabla 6, los sub totales referido al género en los pre-escolares es casi idéntico, con una discreta diferencia del $2.8 \%$ a favor del masculino; en los escolares el favor es para el masculino con el $9.52 \%$ :

En los grandes bloques 9399 sujetos corresponden al masculino y 13858 al femenino, con una ventaja de ésta de 4459 sujetos.

GRAFICO 5: GÉNERO

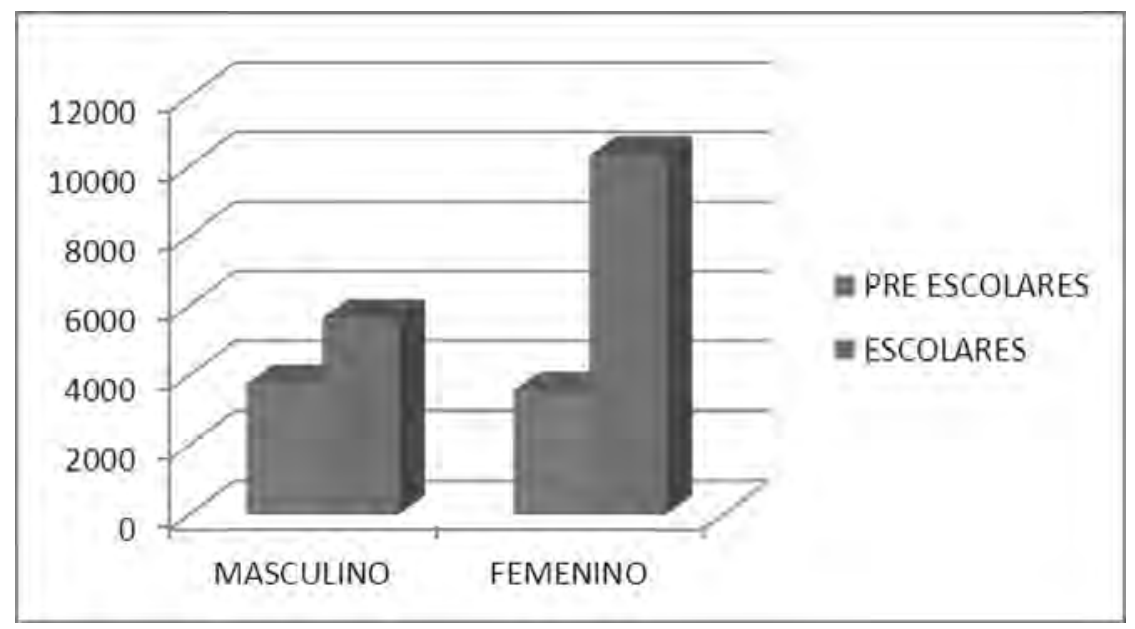

Como se ve en el Gráfico 6, los sub totales referido al género en los pre-escolares es casi idéntico, con una discreta diferencia del $2.8 \%$ a favor del masculino; en los escolares el favor es para el masculino con el $9.52 \%$ :

En los grandes bloques 9399 sujetos corresponden al masculino y 13858 al femenino, con una ventaja de ésta de 4459 sujetos.

TABLA 6: MORBILIDAD Y ESTRATOS SOCIOECONÓMICOS

\begin{tabular}{|c|c|c|c|c|c|c|c|c|c|c|c|c|}
\hline \multirow{2}{*}{ Estratos: } & \multicolumn{2}{|c|}{ Alto } & \multicolumn{2}{c|}{ Medio Alto } & \multicolumn{2}{c|}{ Medio } & \multicolumn{2}{c|}{ Medio Bajo } & \multicolumn{2}{|c|}{ Bajo } & \multicolumn{2}{|c|}{ Totales: } \\
\hline \multirow{2}{*}{ Totales: } & Ss. & $\%$ & Ss. & $\%$ & Ss. & $\%$ & Ss. & $\%$ & Ss. & $\%$ & Ss. & $\%$ \\
\cline { 2 - 13 } & 448 & 3.15 & 2397 & 16.82 & 2695 & 18.91 & 2996 & 21.02 & 5717 & 40.10 & 14253 & 100 \\
\hline
\end{tabular}

Como se ve en la Tabla 6; la morbilidad tiene una curva ascendente desde el estrato socioeconómico Alto con el 3.15\%, como el más bajo porcentualmente, pasa al Medio alto con el $16.82 \%$, siguiendo con el Medio con el $18.91 \%$, continuándose con el Medio bajo con el $21.02 \%$ y culminando con el Bajo con el $40.1 \%$. 
GRAFICO 6: MORBILIDADY ESTRATOS SOCIOECONOMICOS

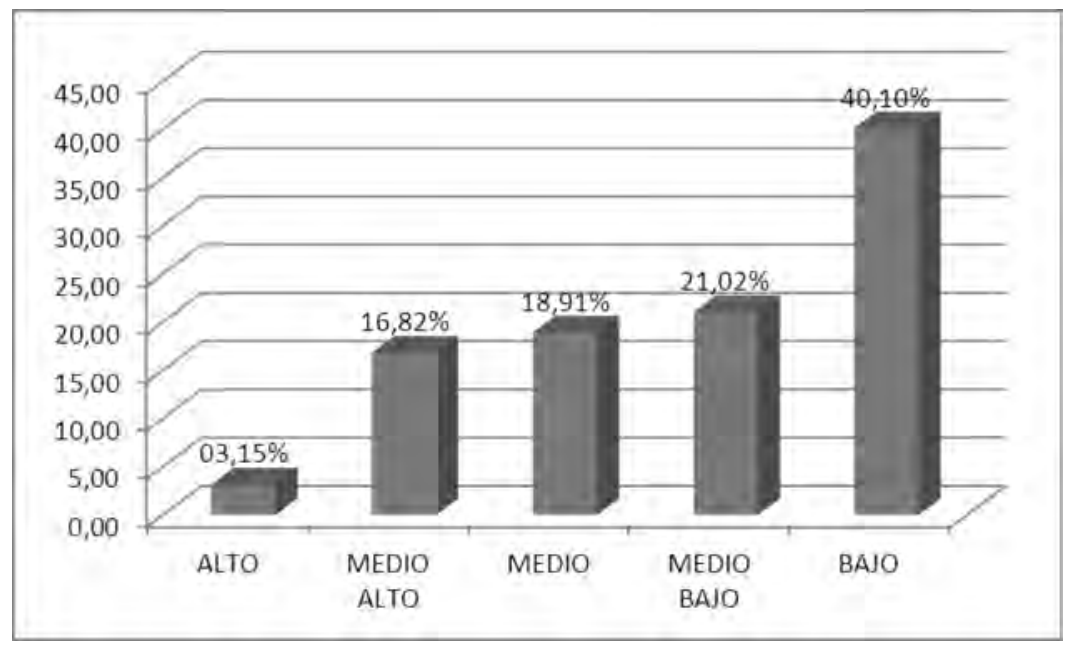

Como se ve en el Gráfico 6; la morbilidad tiene una curva ascendente desde el estrato socioeconómico Alto con el 3.15\%, como el más bajo porcentualmente, pasa al Medio alto con el 16.82\%, siguiendo con el Medio con el $18.91 \%$, continuándose con el Medio bajo con el $21.02 \%$ y culminando con el Bajo con el $40.1 \%$.

\section{Análisis y discusión}

Se tendrá que hacer la salvedad, indicando que, al no existir estudios epidemiológicos sistematizados y ordenados referidos a los trastornos psicopatológicos en los niños menores de 14 años de edad, ni que comprendan a todo el universo psicopatológico que se pueda presentar en las edades denominadas pre-escolar y escolar primaria, tal como el autor lo ha intentado hacer, primero en estudios epidemiológicos sistematizados y coherentes en niños limeños en edades pre-escolares, desde las investigaciones de los años 2000 hasta el 2005, es decir 6 años siguiendo esa línea de investigación, para luego continuar con los niños cuyas edades comprendían a la educación primaria, desde el 2006 hasta el 2010, es decir 5 años en esa línea de investigación referida a los trastornos psicopatológicos; es que a todos estudios de los años del 2000 al 2010, en 11 años, en los cuales el autor realizo 12 investigaciones, tendientes siempre a precisar y clarificar el acontecer mórbido de estos niños; pues los hallazgos que se tienen de otros autores sólo se refieren a cifras aisladas y sin un proceso metodológico de investigación adecuado, por lo difícil de la investigación científica en estos años evolutivos, de cambios violentos y súbitos que se dan en el desarrollo de estos niños.

Una vez hecha esta precisión, pasamos más que a una discusión a un comentario de lo obtenido en este trabajo de condensación, síntesis, agrupamiento de las diversas investigaciones en esta línea que preocupo al autor. 
Así tenemos, referido a los sujetos investigados fueron de 17,611 entre pre-escolares y escolares primarios, lo cual nos muestra un rico universo en cantidad el que se investigó, hallándose como dato importante que en los 2 últimos años, 2009 y 2010 le correspondió el 32.36\% de todo el universo investigado, es decir cerca del tercio, debido a que se rompió el temor de investigar mayor cantidad de sujetos, probablemente por la experiencia, destreza y calidad de los encuestadores, así como por el instrumento que se convirtió en más versátil y de mejor aplicabilidad, pues en esos años se llegó a investigar 3000 y 2700 niños.

El año que se investigó un universo menor fue al comienzo de esta línea de investigación, es decir el año del 2000, donde se tuvieron 600 sujetos con el $3.41 \%$ del universo total y el año del 2009 fue en cual se investigó más sujetos con 3000 y el 17.03\%, es decir más de 5 veces del universo inicial. Se hace más evidente que desde el año del 2006b se comienza a investigar más sujetos, dándonos estos 5 últimos estudios un $56.72 \%$ del universo total en contraste del $43.28 \%$ de las 7 investigaciones anteriores.

La prevalencia o morbilidad encontrada es muy diversa, pero llama la atención el hecho que en el primer estudio del año 2000, se tenga una prevalencia del $92.66 \%$ siendo la más alta de los 12 estudios, se habría debido, probablemente a la dificultad de la encuesta 0 aplicabilidad, a la complejidad del instrumento, como ya se dijo investigar a estas edades del desarrollo es un reto muy difícil y el autor tuvo que buscar un marco teórico que avale la investigación, así como la herramienta más aparente para ese abordaje. En el año del 2006 a se tiene la prevalencia más baja de las investigaciones con el $67.03 \%$, es decir tuvieron que pasar 7 años e igual número de investigaciones para obtener probablemente la cifra más acorde a la realidad de los que acontece con la expresividad mórbida psicopatológica en estos niños, los cuales hasta esta fecha predominaban los niños pre-escolares, pues recién en el año del 2006 se investiga a los niños escolares primarios.

La prevalencia o morbilidad de las 12 investigaciones nos da un promedio de 80.93, es decir, $13.9 \%$ más que la prevalencia del año 2006 a que fue del $67.03 \%$ y $12.73 \%$ menos que la prevalencia del año 2000 que fue del $92.66 \%$.

Referido a los Trastornos psicopatológicos encontrado en las investigaciones, se tiene que buscando uniformarlos de las 12 investigaciones, los Trastornos del Comportamiento con el $22.66 \%$ de promedio es la que predomina, sacándole un $2.92 \%$ de ventaja a los Trastornos del Desarrollo que ocupa el segundo lugar con el $19.74 \%$, seguido de los Trastornos de la Afectividad con el 18.9\%, donde los Trastornos del Comportamiento le saca una ventaja del 3.76\%; entre estos 3 tipos de trastornos psicopatológicos suman el $61.3 \%$ de los trastornos psicopatológicos encontrado en las investigaciones, es decir, más de la mitad de ellos; el Trastorno de la Sexualidad con el 3.4\% es el promedio más bajo de todos los trastornos psicopatológicos, esto podría tener su explicación en el hecho de la edad de los investigados, sobre todo en los pre-escolares.

El encontrar a los Trastornos del Comportamiento como la patología mayoritaria en las investigaciones, y la de mayor promedio nos hace reflexionar que probablemente las inconductas que luego se cristalizan en la adolescencia y juventud, tienen ya sus raíces en 
las edades pre-escolares y escolares primarias, donde los factores podrían ser multicausales, como la dinámica de familia, la falta de enseñanza de valores, y porque no decirlo la pobreza o escases económica.

Cuando analizamos los grados de estudios del universo, se tiene que correspondía a los pre-escolares el $41.44 \%$ y a los escolares primarios el $58.56 \%$, donde hay un mayor número de sujetos investigados de este rango, con una primacía del $17.12 \%$, esta diferencia podría estar inclinando, probablemente hacia el hallazgo de un incremento en alguna alteración psicopatológica, en más o en menos, pero si esto es a los rangos mayores podría no haber un problema mayor, pues se enmascara dentro de los promedios hallados, en el caso que sea a los trastornos psicopatológicos menores, podría estar incidiendo hacia los Trastornos psicosomáticos, Trastornos de los esfínteres o Trastornos sexuales.

En cuanto al género se halla que en los pre-escolares los porcentajes son muy parecidos habiendo solo una discreta diferencia a favor del género masculino con el $2.8 \%$, y en los escolares primarios la ventaja a favor del género masculino es de $9.52 \%$; al hacer el cómputo general de todo el universo se tiene que el género masculino tiene una ventaja del 6.74\%; $3.94 \%$ más en los pre-escolares y $2.78 \%$ en los escolares primarios.

Pudiendo concluirse que las diferencias por género no son altamente significativas, sino guardan una discreta correlación que no debe haber enturbiado los hallazgos de las 12 investigaciones.

Al realizar la correlación entre prevalencia o morbilidad y estratos socioeconómicos, se tiene que hubieron 14, 253 sujetos de la investigación que presentaron indicadores de morbilidad con el $80.93 \%$ del universo investigado que fue de 17,611 sujetos, no habiendo indicadores de morbilidad en el $19.07 \%$ restante, expresando esto que solo esta cifra con 3,358 sujetos no presentaban indicadores de morbilidad, esto se entendió como que estaban exentos de Trastornos psicopatológicos.

Los hallazgos nos muestran una curva ascendente hacia la presencia de indicadores morbilidad partiendo del estrato socioeconómico alto con el $3.15 \%$, seguido por el estrato socioeconómico medio alto con el $16.82 \%$, ascendiendo al estrato socioeconómico medio con el $18.91 \%$ de morbilidad, continuándose con el estrato socioeconómico medio bajo con el $21.02 \%$ para finalizar en la cima con el estrato socioeconómico bajo con el $40.1 \%$ de indicadores de morbilidad, lo que plantea que la diferencia del bajo al alto es de $36.95 \%$, es decir 11 veces más, cifra alarmante por cuanto estaría condicionándose que el estrato socio económico bajo, por diversos motivos: pobreza, hacinamiento, desempleo, desnutrición, enfermedad, etc. genera la posibilidad de presentar indicadores de morbilidad, ya no importando cual trastorno psicopatológico incida, pues habrá de incidir a todos los trastornos psicopatológicos con tan alta tasa de morbilidad, en referencia a los estratos socioeconómicos que la presentan en menos prevalencia. 


\section{Conclusiones y recomendaciones}

Se puede concluir que lo más destacado de la revisión consiste en:

1. El universo investigado abarco a 17, 611 sujetos, entre pre-escolares y escolares primarios.

2. En los años 2009 y 2010 les correspondieron, entre ambos, el $32.36 \%$ del universo total investigado.

3. El año 2000 fue cuando el universo investigado fue menor, con 600 sujetos.

4. El año 2009 cuando el universo investigado fue mayor, con 3000 sujetos.

5. Se evidencia que desde el año 2006b, se comienza a investigar más sujetos, dándonos los 5 últimos estudios el $56.72 \%$ del universo total.

6. En el año 2000 se tiene la prevalencia más alta, con el $92.66 \%$, de todas las investigaciones realizadas.

7. En el año 2006 a , se tiene la prevalencia más baja, con el $67.03 \%$, de todas las investigaciones realizadas.

8. La prevalencia de las 12 investigaciones nos da un promedio del $80.93 \%$.

9. Los trastornos del comportamiento, con el $22.66 \%$ de promedio, son primeros.

10. Los trastornos del desarrollo, con el promedio del $19.74 \%$, ocupa el segundo lugar.

11. Los trastornos de la afectividad, con el promedio del 18.9\%, ocupa el tercer lugar.

12. Los trastornos de la sexualidad, con el promedio del 3.4\%, ocupa el último lugar.

13. Se reflexiona referido a los trastornos del comportamiento, que ya en estas edades tempranas podría estar el inicio de las alteraciones que se evidenciaran en la adolescencia y juventud, de manera, últimamente, tan dramáticas.

14. Hay una discreta ventaja numérica referida a los sujetos investigados que comprenden a los niños de educación primaria, con el $58.56 \%$.

15. En referencia al género hay una discreta diferencia favorable, numéricamente, al masculino en los sujetos pre-escolares y escolares primarios.

16. Se identificaron a 14, 253 sujetos de las investigaciones que presentaron indicadores de morbilidad con el $80.93 \%$.

17. Se identificaron a 3, 358 sujetos de las investigaciones, que no presentaron ningún indicador de morbilidad, pudiendo concluirse que eran exentos de psicopatología, es decir, sanos.

18. En la correlación de prevalencia y estratos socioeconómicos, hay una curva ascendente que parte del estrato socioeconómico alto con el 3.15\% llegando hasta el estrato socioeconómico bajo, con el $40.1 \%$, es decir 11 veces mayor que el inicial.

19. Se sugiere seguir en estas líneas de investigación, las cuales son altamente útiles, en el trabajo de la prevención primaria.

20. Se sugiere que los hallazgos que se han obtenido y se obtengan en futuras investigaciones se den a conocer a los trabajadores de las ciencias de la conducta humana, sobre todo en el campo de la infancia y niñez. 


\section{Referencias bibliográficas}

Oliver PAREDES, Ernesto (1992). Psicopatología del retardo mental fronterizo. Revista de Psicología. Vol. 1. IV. No. 1. Lima: Universidad Ricardo Palma.

Oliver PAREDES, Ernesto (1993). Psicopatología del lenguaje. Revista de Psicología. Vol. IV. No. 2. Lima: Universidad Ricardo Palma.

Oliver PAREDES, Ernesto (1999). La enfermedad mental: Aspectos psicopatológicos y semiológicos. Lima. Libro Amigo.

Oliver PAREDES, Ernesto (2000). Aproximación discreta a la epidemiología de las manifestaciones psicopatológicas infantiles, en Niños preescolares del Cono Sur de Lima Metropolitana. Lima: Centro de Investigación Universidad Ricardo Palma.

Oliver PAREDES, Ernesto (2001a). Acerca de los niños y sus realidades. Lima: Libro Amigo.

Oliver PAREDES, Ernesto (2001b). Aproximación epidemiológica a los trastornos psicopatológicos, en Preescolares de Lima Metropolitana. Lima: Centro de Investigación. Universidad Ricardo Palma.

Oliver PAREDES, Ernesto (2002). Aproximación discreta al estudio de la prevalencia de los trastornos psicopatológicos, en Preescolares de Lima Metropolitana. Lima: Centro de Investigación. Universidad Ricardo Palma.

Oliver PAREDES, Ernesto (2003a). Drogas: Psicopatología del uso, abuso y dependencia a las sustancias psicoactivas. Lima: Libro Amigo.

Oliver PAREDES, Ernesto (2003b). Prevalencia de la salud mental, en Preescolares de Lima Metropolitana. Lima: Centro de Investigación. Universidad Ricardo Palma.

Oliver PARedes, Ernesto (2004a). Prevalencia de la salud mental, en Scientia, Revista del Centro de Investigación de la Universidad Ricardo Palma. Lima: Año VI-N 6, Noviembre, pp. 119-150.

Oliver PAREDES, Ernesto (2004b). Epidemiología del comportamiento anormal, en Niños de Lima Metropolitana. Lima: Centro de Investigación. Universidad Ricardo Palma.

Oliver PAREDES, Ernesto (2005). Aproximación discreta a la prevalencia de los trastornos psicopatológicos específicos, en Niños preescolares de Lima Metropolitana. Lima: Centro de Investigación. Universidad Ricardo Palma.

Oliver PAREDES, Ernesto (2006a). Epidemiología: Indicadores psicopatológicos específicos en niños limeños menores de 6 años de edad. Lima: Inédito.

Oliver PaRedes, Ernesto (2006b). Aproximación a la prevalencia de los trastornos psicopatológicos, en Niños de edad escolar primaria de Lima Metropolitana. Lima: Centro de Investigación. Universidad Ricardo Palma.

Oliver PAREDES, Ernesto (2007a). Psicopatología infantil y epidemiología clínica: Indicadores de trastornos psíquicos. Lima: VIOS S.A.C.

Oliver PARedes, Ernesto (2007b). Aspectos psicopatológicos de los niños en edad escolar primaria. Lima: Centro de Investigación. Universidad Ricardo Palma.

Oliver PARedes, Ernesto (2008). Indicadores generales de trastornos psicopatológicos, en Niños en edad escolar primaria. Lima: Centro de Investigación. Universidad Ricardo Palma. 
Oliver PARedes, Ernesto (2009a). Indicadores específicos de alteraciones psíquicas en niños de edad escolar primaria. Lima: Centro de Investigación. Universidad Ricardo Palma.

Oliver PAREDES, Ernesto (2009b). Breviario terminológico de raíces grecolatinas relacionados a la Medicina, Psiquiatría, Psicopatología, Psicología, Ciencias de la Conducta Humana y Disciplinas Conexas. Lima: Libro Amigo.

Oliver PAREDES, Ernesto (2010). Prevalencia psicopatológica en niños limeños menores de 14 años de edad. Lima: Centro de Investigación. Universidad Ricardo Palma. 